\title{
INFRARED THERMOGRAPHY DIAGNOSTICS OF AIR PERMEABILITY THROUGH BUILDING OPENINGS - ASSESSMENT OF ITS RELIABILITY
}

\author{
Boris BIELEK ${ }^{1}$, Daniel SZABÓ ${ }^{1}$, Jakub ČURPEK ${ }^{1 *}$, Peter SUCHÁNEK ${ }^{2}$, Pavol PANÁČEK ${ }^{3}$
}

\section{Abstract}

The presented paper deals with an analysis of the reliability of a diagnostic method of air permeability through building openings (windows, doors, glass walls) by the application of infrared thermography. A test sample of a plastic double casement window was experimentally tested for air permeability and watertightness in a large pressure chamber. Different levels of the tightness were adjusted between the casement and frame part of the window. Additionally, the window test sample was measured in the large climate chamber with variant air pressure differences. Moreover, the surface temperatures were measured on the window by thermocouples and subsequently compared with images directly taken by infrared thermography. The effect of the increased air infiltration can be detected by infrared thermography but cannot quantify the overall air permeability of the window. The distribution of the exterior window surface temperature is influenced by several factors which should be taken into consideration in the final assessment of the window.
Address

1 Department of Building Construction, Faculty of Civil Engineering STU in Bratislava, Radlinského 11, 81005 Bratislava, Slovakia

2 Rehau, spol. S r.o., Kopčianska 82A, 85000 Bratislava, Slovakia

1 SlovenergoOKNO, o.z., Seberíniho 1, 82103 Bratislava, Slovakia

* Corresponding author: jakub.curpek@stuba.sk

\section{Key words}

- IR thermography,

- Window

- Opening joints,

- Tightness,

- Air permeability.

\section{INTRODUCTION}

Currently, many manufacturers and contractors deal with quite a few complaints about their window products. These problems are mainly based on infrared thermography diagnostics, where the effect of increased air permeability through a window affects the final evaluation. However, for a proper evaluation of this diagnostic method, certain circumstances have to be considered, especially for building openings.

Modern infrared (IR) thermography is a scientific discipline that works with electrooptic systems and devices for the detection and measurement of thermal radiation together with its direct conversion to the surface temperature of the objects investigated. These measurement apparatuses are usually called "infrared" or "thermal" imaging cameras. Their work principle is ensured by a system of thermal display optics, where incidents of infrared radiation are concentrated on a detector. A signal from the optics system is immediately transferred to an electronic screen (a thermogram) on the camera display. A ther- mogram is an electronic picture with a colour scheme, where each colour represents a certain IR wavelength incoming from the surface of the object investigated. IR thermography represents a non-destructive method for the analysis and detection of defective building envelope systems in terms of heat losses, air leakage, and moisture. Many studies have been focused on using IR thermography methods for the detection of air leakages within various building envelope systems (Tiberio and Branchi 2013, Van De Vijver et al. 2014, Dufour et al. 2009, Lerma et al. 2018), where a pressurization fan (Blower Door Test) was used to ensure differences in pressure on both sides (indoor/ outdoor). However, in the IR analysis of a window in the case of air permeability, watertightness has not been investigated in detail yet. In addition, significant deformations of the window occur due to high differences in pressure, together with different air temperatures on each side of the window.

The objective of this study is to focus on the assessment of the reliability of IR thermography in the case of diagnostics of the window structure within a building envelope system. The accuracy of this 


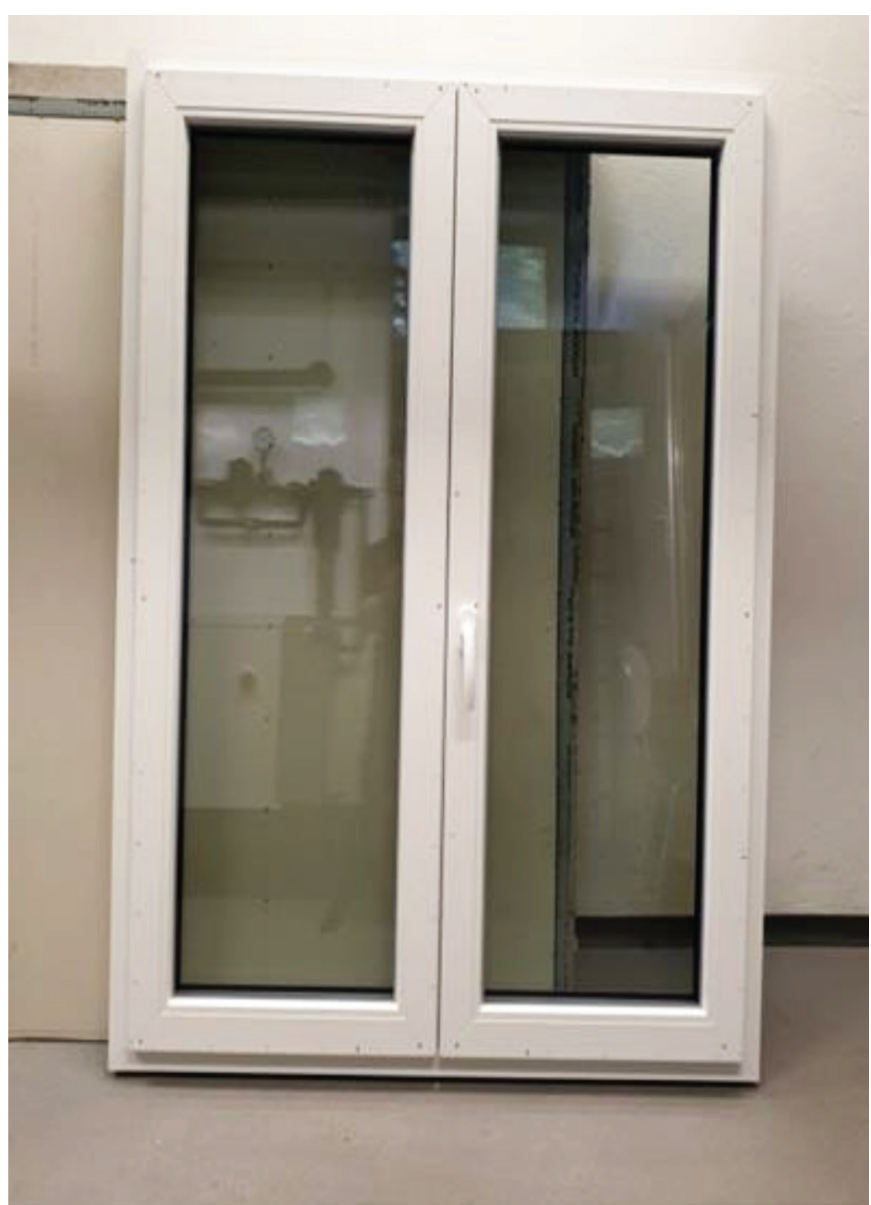

Fig. 1 The tested sample of the plastic double casement window $(1180 \times 1490 \mathrm{~mm})$

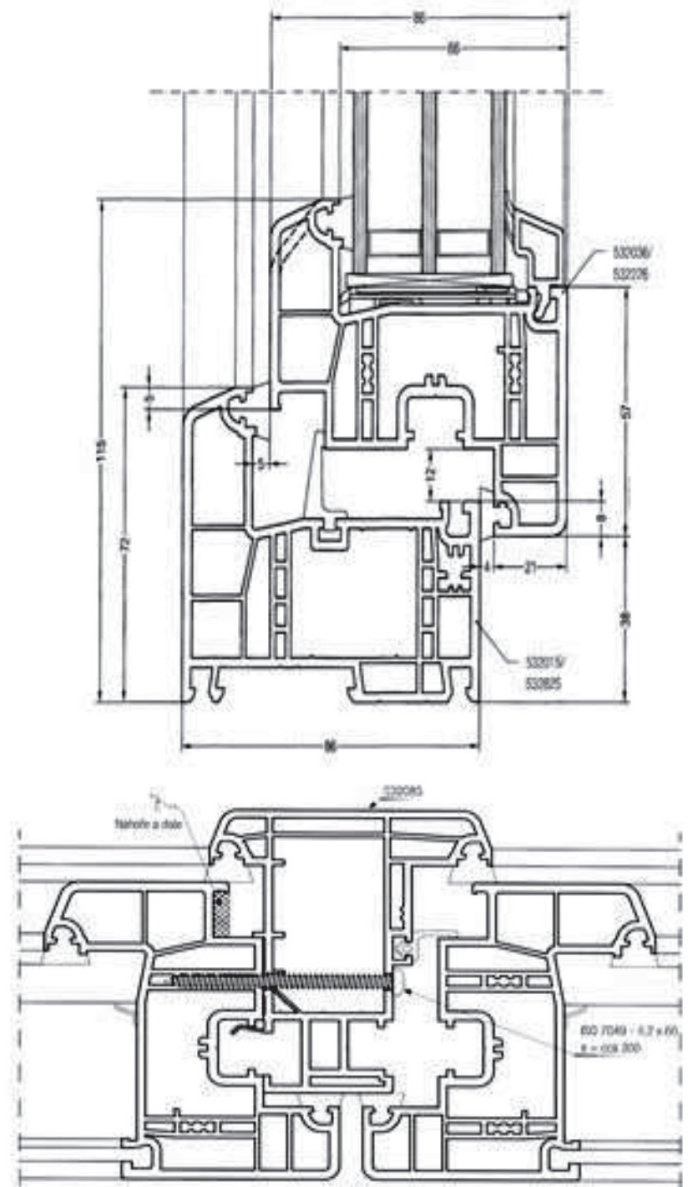

method is questionable, and an adequate assessment should be performed together with a consideration of particular aspects (the tightness of the opening joints, deformations, pressure differences, etc.).

\section{THE WINDOW TEST SAMPLE}

An experimental analysis was performed on a sample of a plastic double casement window $(1180 \times 1490 \mathrm{~mm})$ without a middle mullion, where one part on a side is hung inwards and the other one is tilted and turned inwards (Fig. 1). The window frames used were from the REHAU Geneo ( $86 \mathrm{~mm}$ profile) system, which is a RAU-FITPRO fibre composite material. The windowpane system is composed of closed insulation units, a three glass 4-16-4-16-4 mm system, and a $2 x$ Planitherm with argon. The opening joints are sealed on three levels: with an inside seal (a wind barrier), a middle seal (closure of the decompression cavity), and an outside seal. The window fittings are based on the GU system, which goes around the whole window's perimeter. The tightness of the opening joints between the casement and frame part of the window was modified at three positions (maximum, medium, minimum) by the adjustment of the window fittings. Accordingly, the compression of the opening joints was modified, which therefore changed the air permeability of the window.

\section{EXPERIMENTAL LABORATORY ANALYSES}

The window test sample was measured at three levels of the tightness of the opening joints in the Laboratory of Building Physics and Structures, Faculty of Civil Engineering STU in Bratislava. Two ex- perimental measurement facilities were used in the research: a large pressure chamber and a large climate chamber. Various physical parameters were investigated: the air permeability, the velocity of the air flow through the opening joints at several differences in air pressure, deformations of the window sash in relation to the frame due to variable differences in pressure, watertightness (in the large pressure chamber), the heat transfer coefficient, and the surface temperatures (in the large climate chamber).

\subsection{Quantification of the air permeability through the window}

The window sample was tested in the large pressure chamber under various pressure differences of $\Delta p=10,30,100,300,450$ and $600 \mathrm{~Pa}$, together with the three levels of the tightness of the opening joints between the casement and frame part of the window. The air permeability of the window sample was measured according to STN EN 1026 (2016). The classification is based on a comparison of the air permeability of the window test sample related to the overall area (Fig. 2, left) and on the air permeability related to the length of the opening joints (Fig. 2, right) (STN EN 12207, 2017). The reference values of the air permeability for the overall window area and the length of the opening joint are defined at a reference test pressure of $100 \mathrm{~Pa}$ (STN EN 12207, 2017).

The local air permeability through the opening joints was measured by an anemometer. The local velocity of the infiltrating air was measured at 65 points through the overall perimeter of the opening joints (Fig. 3). 

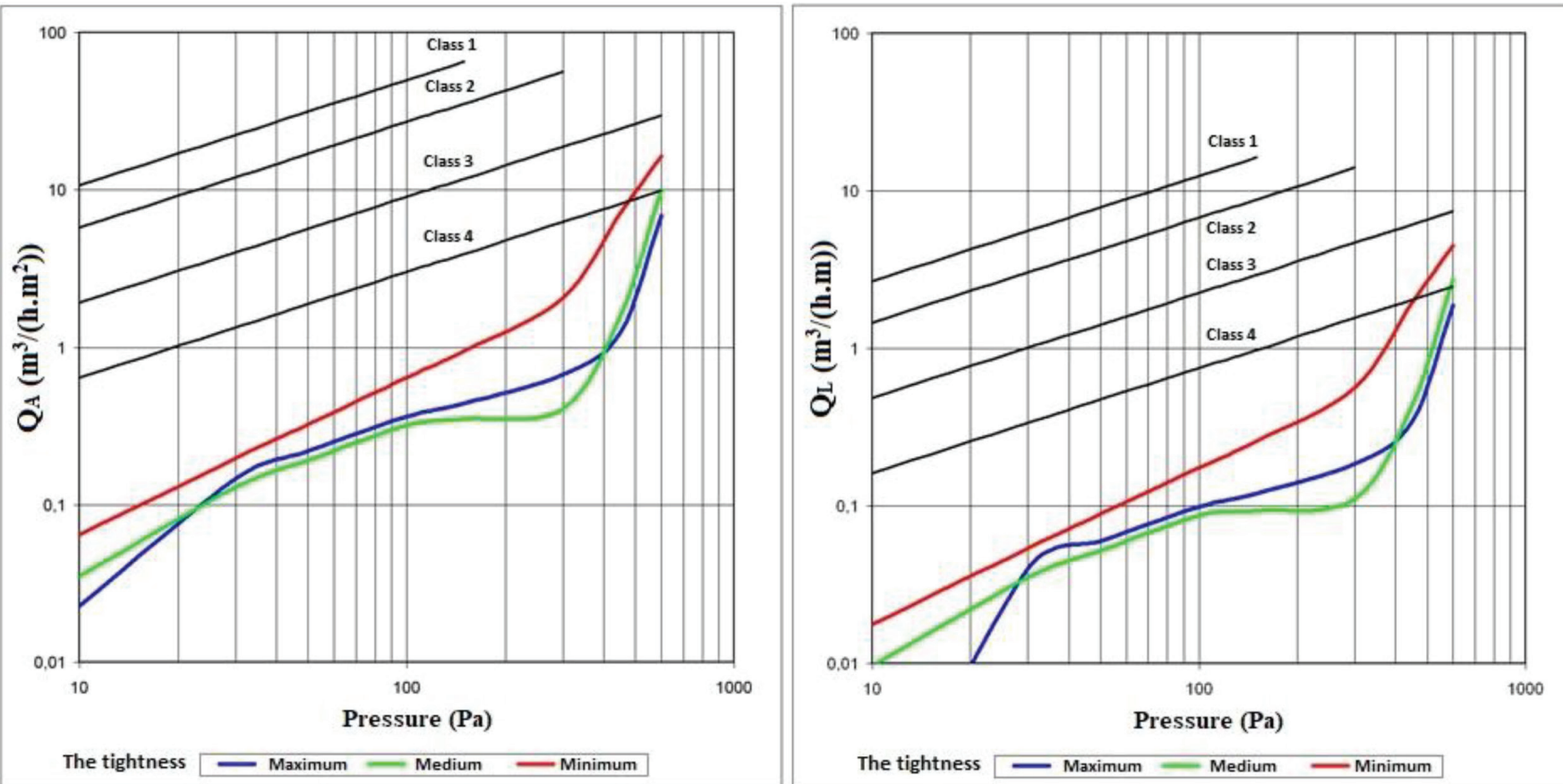

Fig. 2 Diagrams of the air permeability of the window sample relative to the measured window area (left) and to the length of the opening joints (right), at three different levels of tightness.

\subsection{Quantification of the window deformations}

The differences between the air pressure on both sides (interior and exterior) of the window cause significant deformations of the window and directly influence its overall tightness. The movement of the air flow through the opening joints shifts, depending on the deformations (the strength and stability of the window frame parts). In this case, twelve laser sensors were used for the measurement

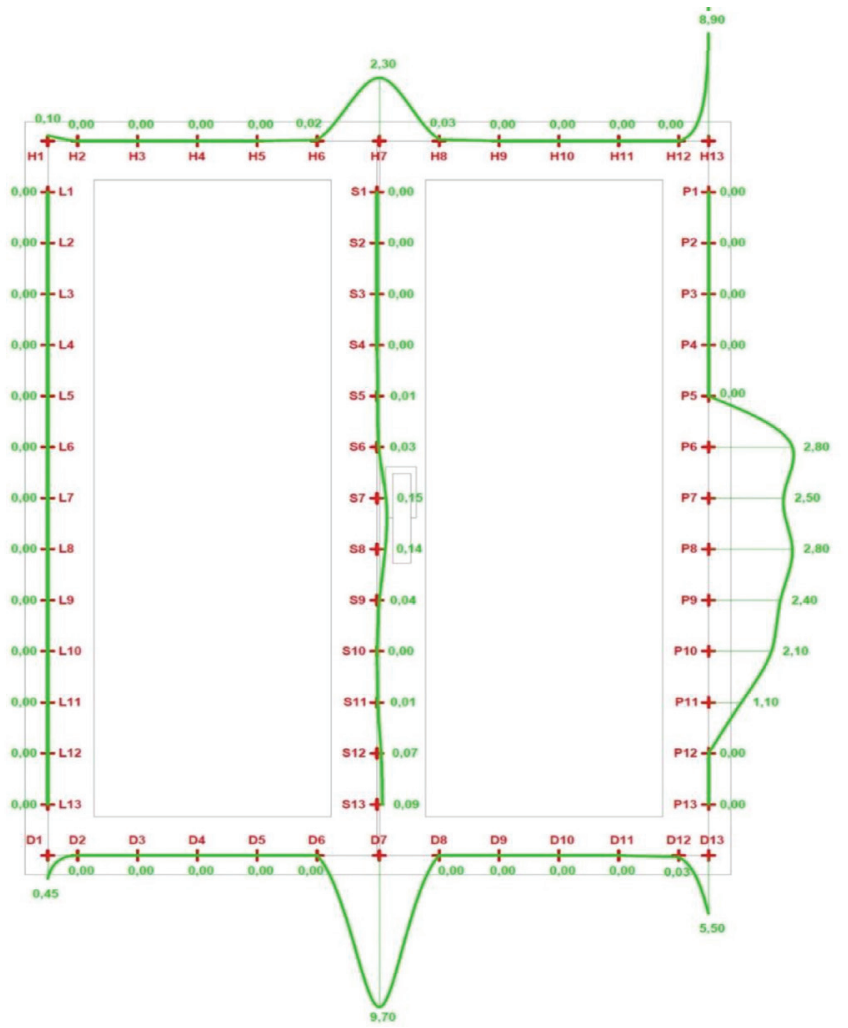

Fig. 3 Diagram of the local velocity of the infiltrated air $(\mathrm{m} / \mathrm{s})$ through the overall perimeter of the opening joints at $\Delta p=600 \mathrm{~Pa}$ at a medium level of tightness. of the window frame deformations at three levels through the window's height (Fig. 4) and under the various differences in pressure of $\Delta p=10,30,100,300,450$ and $600 \mathrm{~Pa}$, together with the three levels of tightness of the opening joints between the casement and the frame part of the window.

\subsection{Quantification of the watertightness of the window}

The ability of the window test sample to resist water penetration was measured under the test conditions according to STN EN 1027

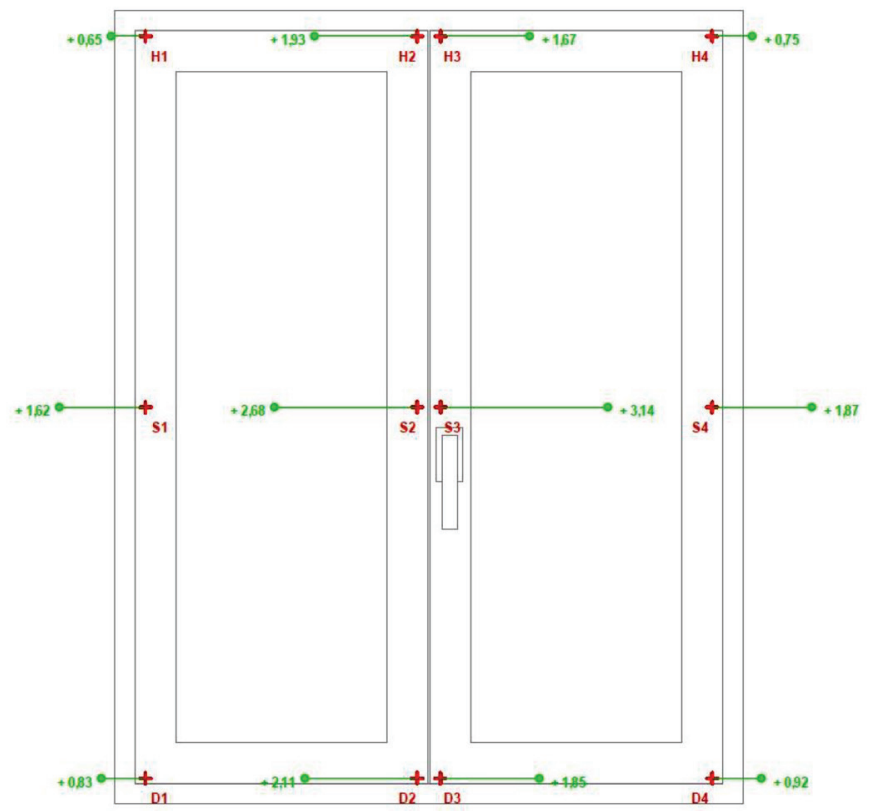

Fig. 4 Diagram of the window deformation ( $\mathrm{mm}$ ) at $\Delta p=600 \mathrm{~Pa}$ at a medium level of tightness. 


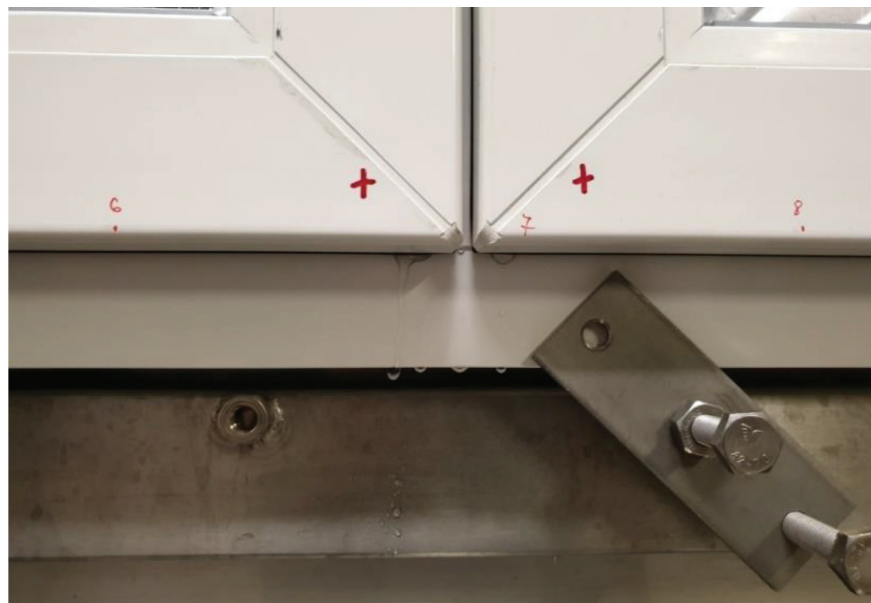

Fig. 5 The effect of losing the ability of the window sample to resist water penetration

(2016) in the large pressure chamber at the three levels of tightness of the opening joints between the casement and frame part of the window. The effect of losing the ability of the window sample to resist water penetration occurred under certain levels of air pressure differences $(\Delta p=50,100,150 \mathrm{~Pa})$ depicted in Fig. 5 .

\subsection{Summary of the experimental results in the large pressure chamber}

The overall experimental results of the physical parameters of the investigated window are summarized in Tab. 1 at the three different levels of tightness. According to the results, the level of tightness of the opening joints between the casement and frame part of the window significantly influences the air permeability and the deformations as well as the watertightness. Considering that the heat transfer coefficient is determined to be at zero value of the difference in air pressure, the influence of the level of tightness does not have an effect on the coefficient quantification. The experimental results revealed that the level of tightness considerably influences the window's air permeability, where cold infiltrated air rapidly influences the interior surface temperature (a decreasing effect). An adjustment of the level of tightness is crucial for the interior surface temperature of a window.

\subsection{IR thermography measurements of the sample}

The main goal of the presented paper is based on an assessment of the reliability of the IR thermography method for an analysis of window surface temperatures. The window test sample was placed in and measured by the large climate chamber's test equipment, where the thermography was performed. The thermograms were taken using a Flir T620 camera with a high resolution $(640 \times 480$ pixels $)$ at different boundary conditions together with various window adjustments:

- at a different outside air temperature in the cold chamber $\left(\theta_{a e}=+2^{\circ} \mathrm{C}, \theta_{a e}=-11^{\circ} \mathrm{C}\right)$ and at temperature $\theta_{a i}=+22^{\circ} \mathrm{C}$ in the warm chamber;

- at three differences in air pressure between the cold and warm chamber, $\Delta p=10 \mathrm{~Pa}, 50 \mathrm{~Pa}, 100 \mathrm{~Pa}$;

- at three levels of tightness of the opening joints between the casement and frame part of the window.

The colour spectrum of the IR camera was set up in a temperature range from $+8^{\circ} \mathrm{C}$ to $+23^{\circ} \mathrm{C}$, due to the subsequent mutual comparisons of the measurements.

\subsubsection{Analysis of the thermograms and their confrontation with the experiments}

Various circumstances have to be taken into account with the in-situ process of the IR thermography method in the case of window structure diagnostics. The experimental measurements using the laboratory equipment in comparison with the thermograms revealed nu-

Tab. 1 Physical parameters of the investigated window obtained by the laboratory experimental measurements at three levels of tightness of the opening joints between the casement and frame part of the window

\begin{tabular}{|c|c|c|c|c|}
\hline \multirow{2}{*}{ Test method } & \multirow{2}{*}{ Parameter } & \multicolumn{3}{|c|}{ Level of the tightness } \\
\hline & & maximum & medium & minimum \\
\hline \multirow{3}{*}{ Air permeability } & Class of air permeability according to STN EN 12207: 2017 & 4 & 3 & 3 \\
\hline & Volume of air flow rate at $\Delta p=600 \mathrm{~Pa} Q_{o b, 600}\left(\mathrm{~m}^{3} / \mathrm{h}\right)$ & 12.07 & 17.59 & 28.99 \\
\hline & Max. velocity of the infiltrating air flow through open joints $v_{\text {inf }}(\mathrm{m} / \mathrm{s})$ & 6.6 & 9.7 & 30.0 \\
\hline Deformation & Max. measured deformation of the window sash in relation to the frame $(\mathrm{mm})$ & 0.93 & 1.44 & 1.23 \\
\hline \multirow{2}{*}{ Watertightness } & Limit of watertightness of the window at pressure difference $\Delta p(\mathrm{~Pa})$ & 150 & 100 & 50 \\
\hline & Class of watertightness according to STN EN 12208: 2001 & $4 \mathrm{~A}$ & $3 \mathrm{~A}$ & $2 \mathrm{~A}$ \\
\hline \multirow{4}{*}{$\begin{array}{l}\text { Building heat } \\
\text { transfer }\end{array}$} & Standardized heat transfer coefficient of the window $U_{s t}\left(\mathrm{~W} /\left(\mathrm{m}^{2} . \mathrm{K}\right)\right)$ & 1.05 & 1.06 & 1.09 \\
\hline & $\begin{array}{l}\text { Min. int. surface temperature at point } 3 \\
\theta_{s i 3}\left({ }^{\circ} \mathrm{C}\right),\left(\theta_{a e}=-11^{\circ} \mathrm{C}, \Delta p=0 \mathrm{~Pa}\right)\end{array}$ & 13.01 & 12.88 & 10.36 \\
\hline & $\begin{array}{l}\text { Min. int. surface temperature at point } 3 \\
\theta_{s i 3}\left({ }^{\circ} \mathrm{C}\right),\left(\theta_{a e}=-11^{\circ} \mathrm{C}, \Delta p=50 \mathrm{~Pa}\right)\end{array}$ & 11.32 & 11.13 & 9.26 \\
\hline & $\begin{array}{l}\text { Min. int. surface temperature at point } 3 \\
\theta_{s i 3}\left({ }^{\circ} \mathrm{C}\right),\left(\theta_{a e}=-11^{\circ} \mathrm{C}, \Delta p=100 \mathrm{~Pa}\right)\end{array}$ & 10.80 & 10.61 & 5.42 \\
\hline
\end{tabular}


merous findings which should be considered for a proper evaluation of the IR thermography and window assessment:

- at a zero difference in air pressure of $\Delta p=0 \mathrm{~Pa}$, there is no distinction between the window thermograms with different levels of tightness of the opening joints between the casement and frame part (Fig. 6). There is no air infiltration through the open joints at a zero difference in air pressure (no wind effect);

- a continuous increase in the difference in air pressure $\Delta p$ (from 0 to $100 \mathrm{~Pa}$ ) caused significant changes in the window thermograms - the infiltrated cold air decreased the interior surface temperature of the window (Fig. 7);

\section{A}

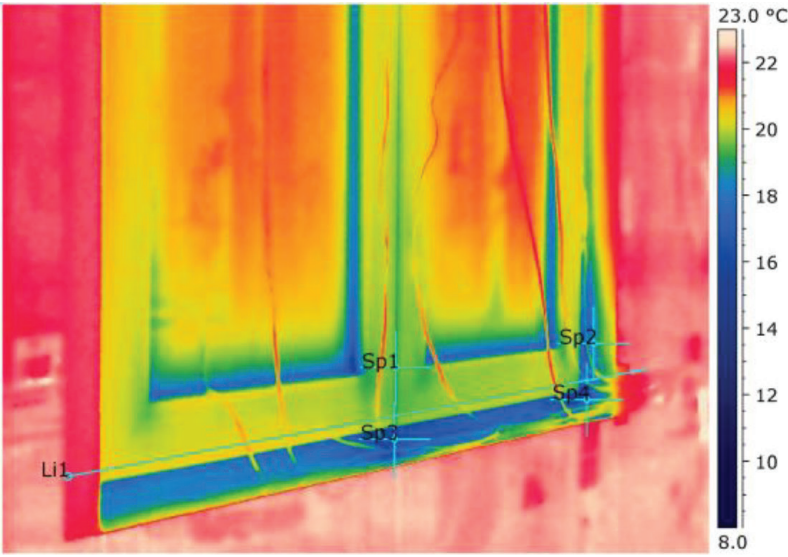

- a comparison between the two thermograms, where the levels of tightness were adjusted at the minimum and maximum positions, revealed no significant changes in the same boundary conditions (Fig. 8). However, in this case, the air flow volumes had different values $\left(Q_{\text {ob,min.tight, 100Pa }}=1.126 \mathrm{~m}^{3} / \mathrm{h}\right.$ and $Q_{\text {ob,max.tight, } 100 \mathrm{~Pa}}=0.64 \mathrm{~m}^{3} / \mathrm{h}$ ) and did not influence the above-mentioned thermograms;

- no distinction between the window thermograms in the case of the same level of the tightness and at equal values of $\Delta p$, $\theta_{a i}$ with a combination of different exterior temperatures $\theta_{a e}$, $\left(\theta_{a e}=-11^{\circ} \mathrm{C} \mathrm{a} \theta_{a e}=+2{ }^{\circ} \mathrm{C}\right)$, (Fig. 9);

B

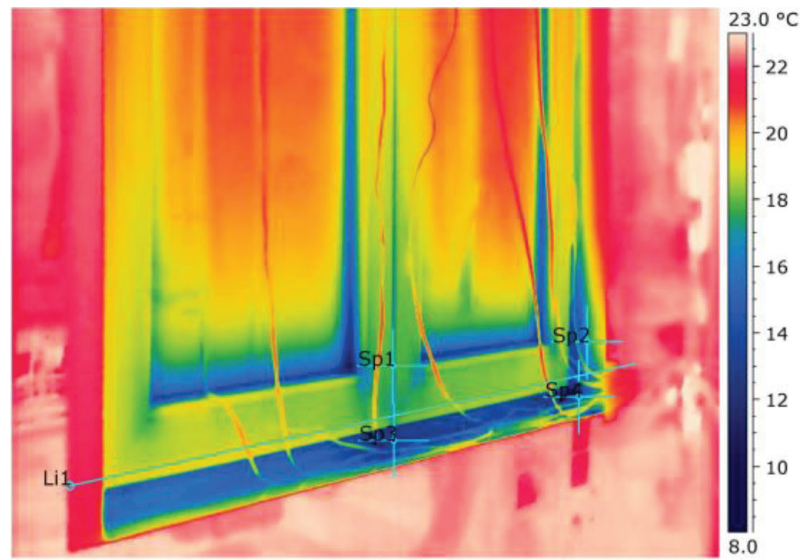

Fig. 6 Thermograms of the window revealed no differences in the case of $\Delta p=0$ Pa at different levels of the adjusted tightness, $A-$ minimal level of the tightness, $B$-medium level of the tightness. $\theta_{a i}=+22^{\circ} \mathrm{C}, \theta_{a e}=-11^{\circ} \mathrm{C}$

A

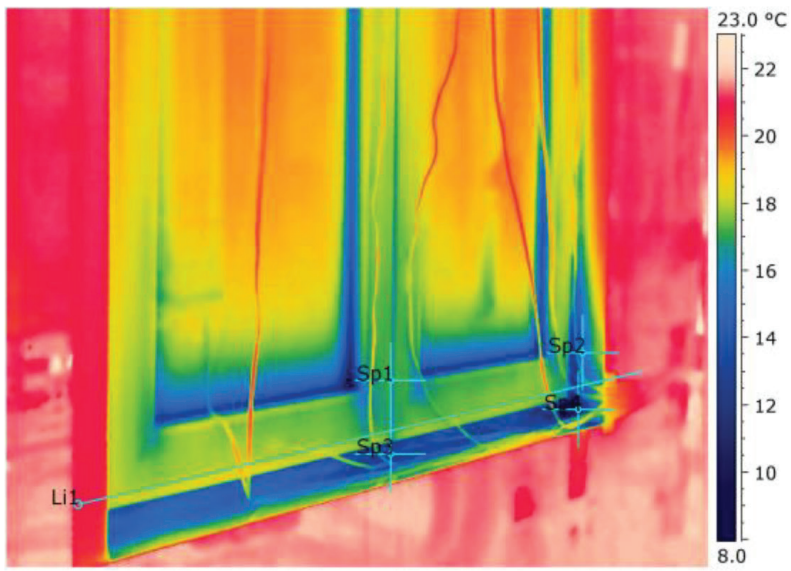

C

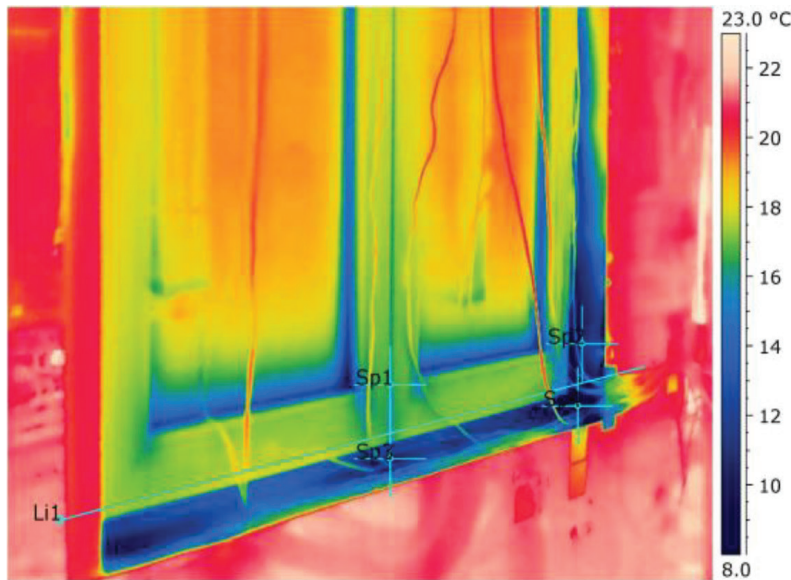

B

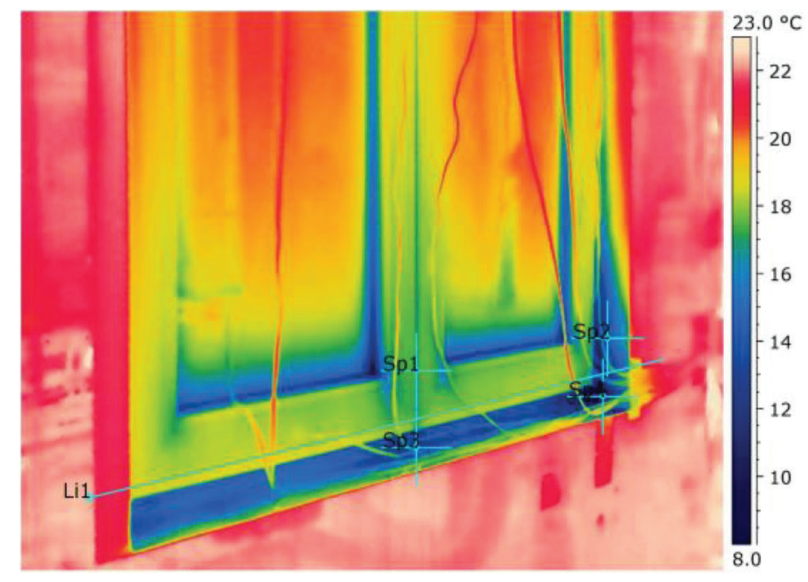

D

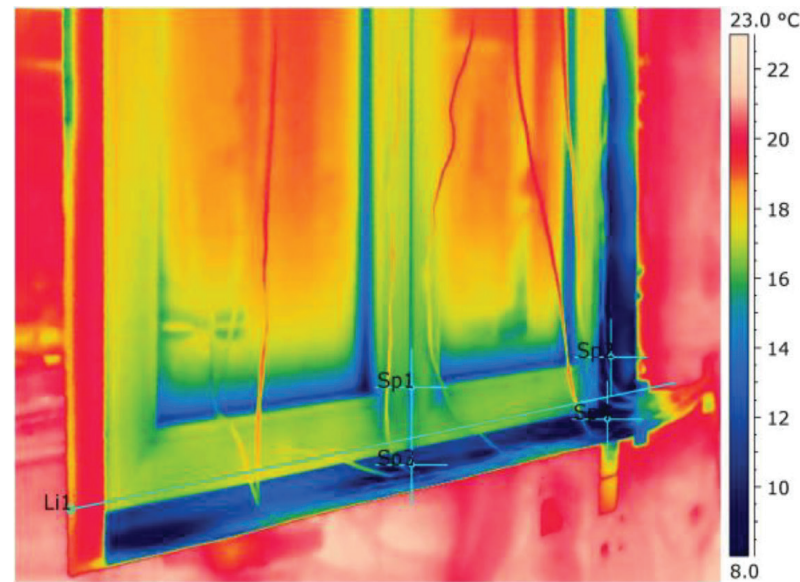

Fig. 7. Thermograms of the window revealed significant differences through a continuous increase of $\triangle p$ from $0 P a$ to $100 P a$. A - $\triangle p=0 P a$, $B-\Delta p=10 \mathrm{~Pa}, \mathrm{C}-\Delta p=50 \mathrm{~Pa}, \mathrm{D}-\Delta p=100 \mathrm{~Pa} . \theta_{a i}=+22^{\circ} \mathrm{C}, \theta_{a e}=-11^{\circ} \mathrm{C}$, medium level of tightness 
A

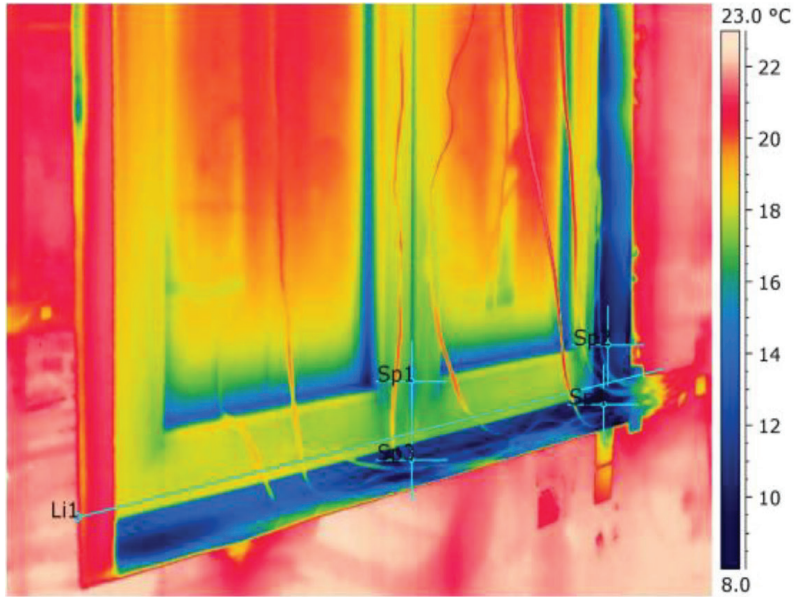

B

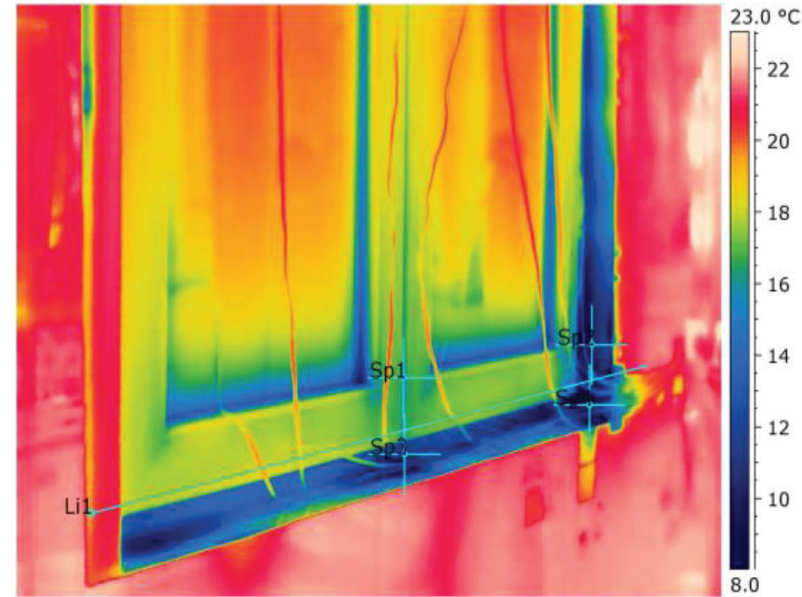

Fig. 8 The thermograms of the window are very similar at the minimum and maximum levels of the tightness together with the same boundary conditions. A - the window with an adjusted minimum level of the tightness, $B$ - the window with an adjusted maximum level of the tightness. $\theta_{a i}=+22^{\circ} \mathrm{C}, \theta_{a e}=-11^{\circ} \mathrm{C}, \Delta p=100 \mathrm{~Pa}$

A

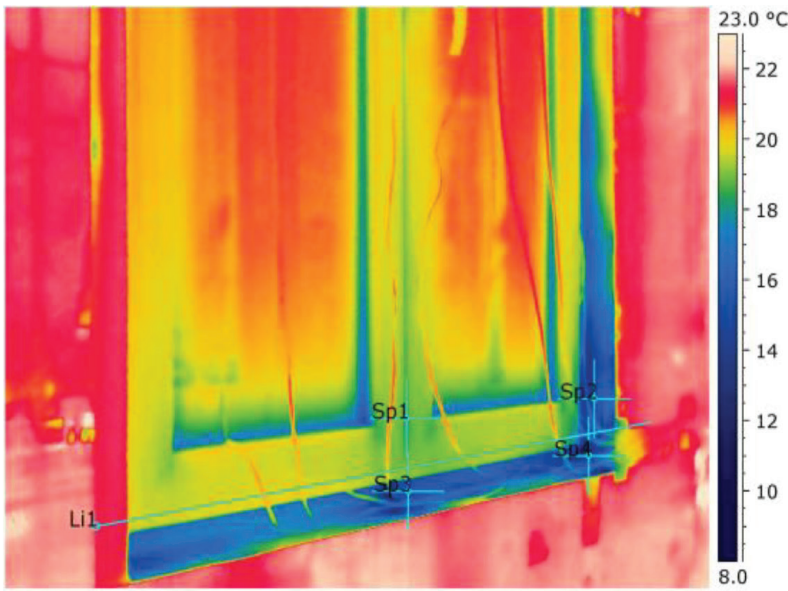

B

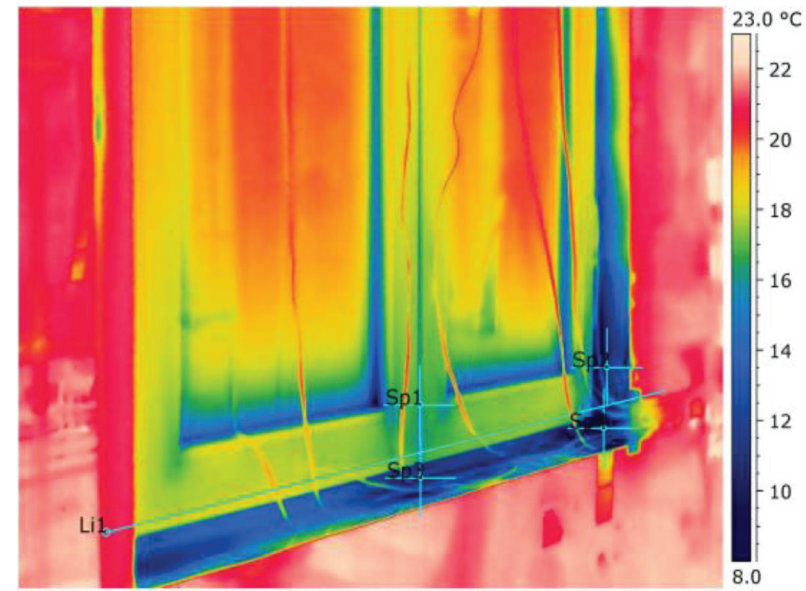

Fig. 9 The thermograms of the window are very similar at the same levels of tightness, $\Delta p$ and $\theta_{a,}$, but at different values of $\theta_{a e^{\circ}}$ $A-\theta_{a e}=+2{ }^{\circ} \mathrm{C}, \mathrm{B}-\theta_{a e}=-11^{\circ} \mathrm{C} . \theta_{a i}=+22^{\circ} \mathrm{C}, \Delta p=50 \mathrm{~Pa}$, adjusted minimum level of the tightness

A

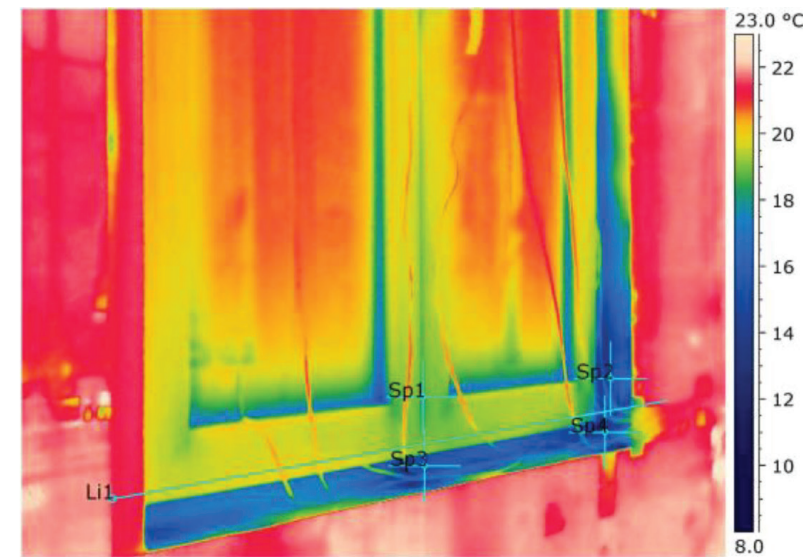

B

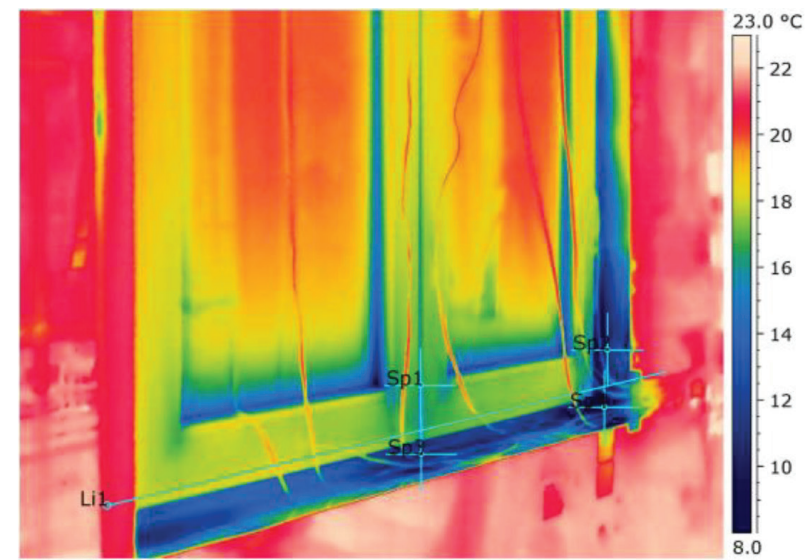

Fig. 10 The thermograms of the window are very similar in the two cases - at the maximum level of the tightness together with the higher values of $\Delta p$, and at the minimum level of the tightness together with the lower values of $\Delta p$. A-the window with the adjusted minimum level of the tightness, $\triangle p=50 \mathrm{~Pa}, \mathrm{~B}$ - the window with the adjusted maximum level of the tightness, $\Delta p=100 \mathrm{~Pa} . \theta_{a i}=+22^{\circ} \mathrm{C}, \theta_{a e}=-11^{\circ} \mathrm{C}$

- there were very similar thermograms of the window with the maximum level of the tightness at the higher values of $\Delta p$ and of the window with the minimum level of the tightness at the lower values of $\Delta p$, at the same boundary conditions (Fig. 10). The mutual comparison of the air flow volumes of the above-mentioned windows $\left(Q_{o b \min \text { tight } 50 \text { Pa }}=0.572 \mathrm{~m}^{3} / \mathrm{h}\right.$ a $Q_{o b \max \text { tight 100Pa }}=$ $0.64 \mathrm{~m}^{3} / \mathrm{h}$ ) revealed different airtightness classes (the window with the minimum level of the tightness (Class 3 ) and the win- 
dow with the maximum level of tightness (Class 4) according to STN EN 12207 (2017)). These windows had very similar air flow volumes $Q_{o b}$ at different levels of $\Delta p$ (50 and $100 \mathrm{~Pa}$ ); therefore, their thermograms did not show significant distinctions. Accordingly, windows with different levels of air permeability can have very similar thermograms at different levels of $\Delta p$ (at the maximum level of the tightness together with the higher levels of $\Delta p$ and at the minimum level of tightness together with the lower levels of $\Delta p$ ).

- no significant distinctions in the window thermograms in the cases of the maximum level of the tightness at the higher values of $\theta_{a e}$, and the minimum level of the tightness at the lower values of $\theta_{a e}$-Figure 11. The air flow volumes through the two above-mentioned cases are different $\left(Q_{\text {ob,min.tight, I00Pa }}=1.126 \mathrm{~m}^{3} / \mathrm{h}\right.$ a $Q_{\text {ob,max.tight, } 100 \mathrm{~Pa}}=0.640 \mathrm{~m}^{3} / \mathrm{h}$ ). Accordingly, these windows are in different airtightness classes, i.e., the window with the minimum level of tightness (Class 3 ) and the window with the maximum level of tightness (Class 4) according to STN EN 12207 (2017). In spite of this, the thermogram of the tighter window with cooler infiltrating air is very similar to the thermogram of the less tight window with the warmer infiltrating air.

- the surface temperatures are influenced by the infiltrating air through the connections/joints within the building envelope structures and are visualized on the thermograms by ideal spike

A

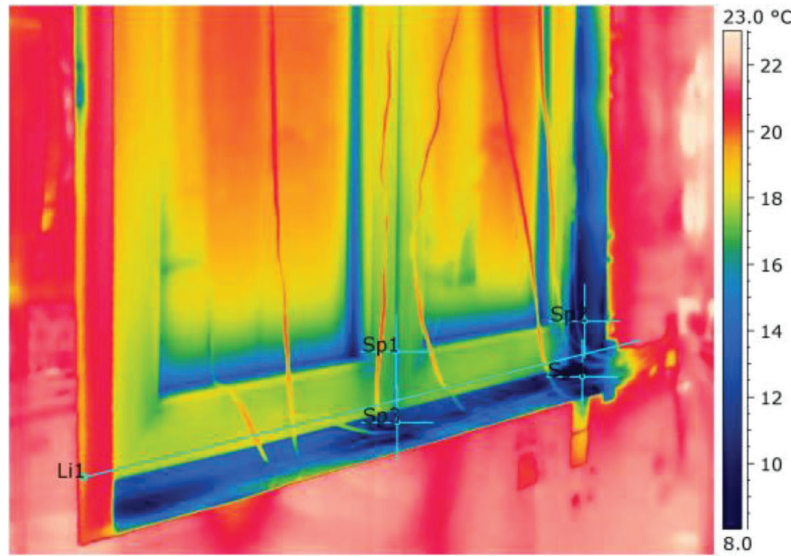

nozzle shapes. The investigated window was classified in airtightness classes 3 and 4; accordingly the effect of the infiltrating cool air through the opening joint was not observed on the thermograms, except for the one location, i.e., the right bottom corner of the window, where the many cables of the Pt100 surface temperature sensors are located (Fig. 12).

- the accuracy of the surface temperature measurement by the Flir T620 thermal imaging camera was in a range of $-0.91{ }^{\circ} \mathrm{C}$ to $+1.19^{\circ} \mathrm{C}$ (approximately $\pm 1^{\circ} \mathrm{C}$ ) in comparison with the calibrated Pt100 thermal sensors during the laboratory experimental measurements. Generally, there are a lot of cameras with different imaging resolutions; it is therefore not possible to exactly determine the surface temperatures. This method is only used for the approximate layout of the surface temperatures.

\section{CONCLUSIONS}

According to the above-mentioned analysis of the window thermograms and their confrontation with the physical parameters of the window obtained by the laboratory experimental measurements, it is possible to state the following conclusions:

- infrared thermography is a modern diagnostic method for the detection of defects or problems within a building envelope

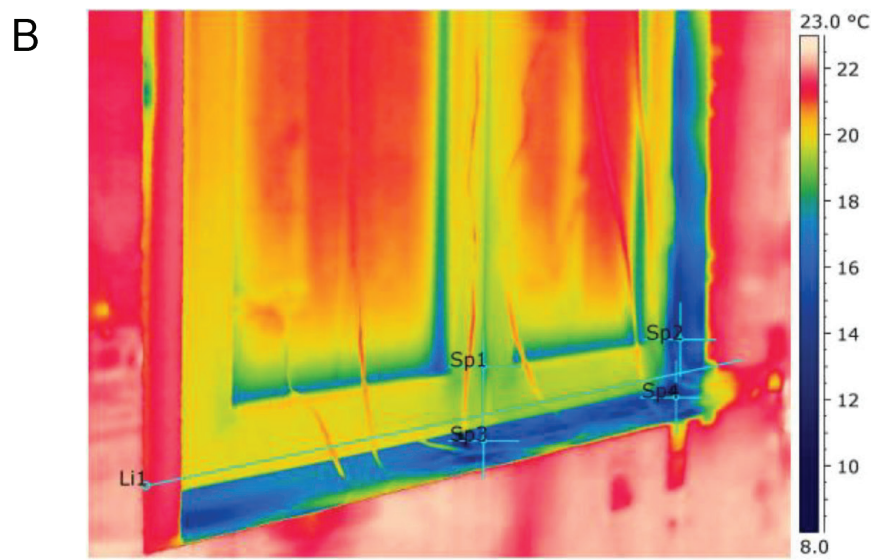

Fig. 11 The thermograms of the window are very similar in two cases - at the maximum level of tightness together with the higher values of $\theta_{a e^{\prime}}$ and at the minimum level of tightness together with the lower values of $\theta_{a e}$. A-the window with the adjusted maximum level of the tightness, $\theta_{a e}=-11^{\circ} \mathrm{C}, \mathrm{B}$ - the window with the adjusted minimum level of the tightness, $\theta_{a e}=+2{ }^{\circ} \mathrm{C} . \theta_{a i}=+22^{\circ} \mathrm{C}, \Delta p=100 \mathrm{~Pa}$

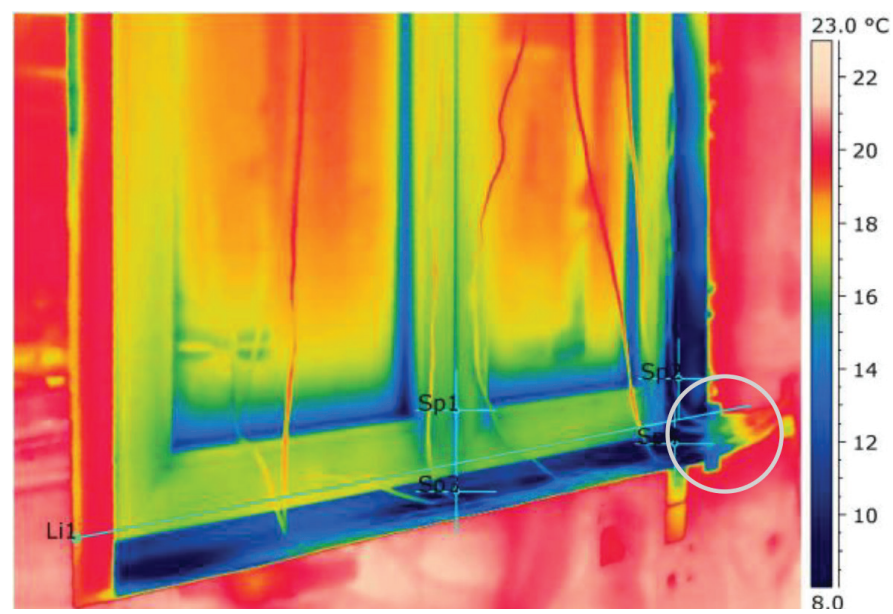

Fig. 12 Thermogram of a window with the effect of infiltrating cool air visualized by the ideal spike nozzle shape in the window's corner. The adjusted medium level of the tightness $\theta a i=+22^{\circ} \mathrm{C}$, $\theta a \mathrm{e}=$ $-11^{\circ} \mathrm{C}, \Delta \mathrm{p}=100 \mathrm{~Pa}$

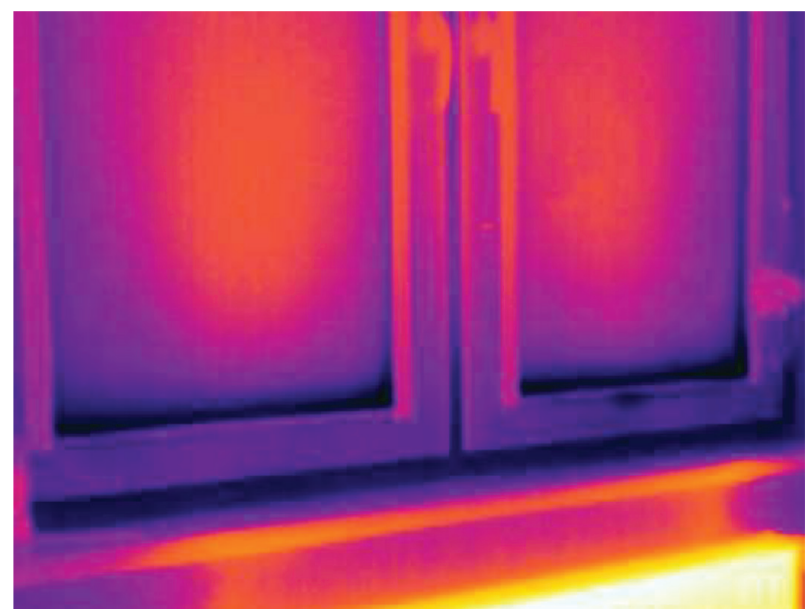

Fig. 13 Thermogram of a window with the effect of the heating unit placed under the windowsill 
system. However, this method cannot exactly quantify these problems, but instead represents a significant part of building diagnostics, mainly due to its speed of detection and the objectivity of potential risks;

- a thermal imaging camera does not serve for the exact quantification of surface temperatures, but for the orientational and visually well-arranged distribution of surface temperatures on a surface;

- if the surface temperatures are significantly influenced by infiltrating air through the connections and joints within building envelope structures, then the air can be tracked by ideal spike nozzle shapes on a thermogram (usually in the case of a high rate of air flow volume). On the other hand, in the case of a low rate of air flow volume, the ideal spike nozzle shapes cannot be observed on a thermogram, but that does not mean that the infiltrating air cannot influence the interior surface temperatures;

- thermograms are particularly influenced by outside boundary conditions (the tighter window at a higher value of $\Delta p$ has a similar thermogram as the less tight window at a lower value of $\Delta p$; the tighter window at a lower value of $\theta_{a e}$ has a similar thermogram as the less tight window at a higher value of $\theta_{a e}$, etc.). Accordingly, it is necessary to specify certain objects in the protocol of the thermal imaging measurements: the date and time of the re- alization, the type of thermal imaging camera and its objectives, the adjusted emissivity on camera, the exterior air temperature $\theta_{a e}$, the relative humidity of the exterior $\varphi_{e}$, the interior air temperature $\theta_{a i}$, the relative humidity of interior $\varphi_{i}$, and the differences in air pressure on both sides of the measured window $\Delta p$;

- the distribution of the exterior surface temperature scanned by a thermal imaging camera is influenced (except for the effects of heat transmission or air infiltration) by other factors, i.e., direct solar radiation, heating units (convective, radiative), the window installation, the window's thermophysical properties, the movement of the air flow in the building, the vertical distribution of the interior air temperatures, etc. The effect of the heating unit on the window surface temperatures is depicted in Fig. 13.

- a window thermogram can detect places or locations with increased air infiltration; however, it is not possible to quantify the air permeability of the window. The thermogram cannot be a relevant document for a window reclamation due to its air permeability.

- if the window thermogram detects increased air infiltration, then additional diagnostic methods are needed for evidence of the excessive effect of air infiltration through the window, for example, measurement of the air flow through the opening joints or a blower-door test.

\section{Acknowledgement}

This work was supported by the Slovak Research and Development Agency under Contract No. APVV-16-0126 and by Scientific Grant Agency MŠVVŠ SR and SAV under VEGA 1/0113/19.

\section{REFERENCES}

Dufour, M. B., Derome, D., Zmeureanu, R. (2009) Analysis of thermograms for the estimation of dimensions of cracks in building envelope. Infrared Physics and Technology, Vol. 52, pp. 70-78.

Fluke (2009) Introduction to Thermography Principles. Amer Technical Pub, ISBN 10: 0826915353.

Lerma, C., Barreira, E., Almeida, R.M.S.F. (2018) A discussion concerning active infrared thermography in the evaluation of building air infiltration. Energy and Buildings, Vol. 168, pp. 56-66.

STN EN 1026 : 2016 Windows and doors, Air permeability, Test method.

STN EN 12207 : 2017 Windows and doors, Air permeability, Classification.

STN EN 1027 : 2016 Windows and doors, Watertightness, Test method.

STN EN 12208 : 2001 Windows and doors, Watertightness, Classification.
STN EN ISO 12567-1: 2011 Thermal performance of windows and doors. Determination of thermal transmittance by the hot-box method. Part 1: Complete windows and doors. (ISO 125671:2010)

Tiberio, A. J., Branchi, P. (2013) A study of air leakage in residential buildings. International Conference on New Concepts in Smart Cities: Fostering Public and Private Alliances (SmartMILE), Gijon, 2013, pp. 1-4.

Van De Vijver, S., Steeman, M., Carbonez, K., \& Van Den Bossche, N. (2014) On the use of infrared thermography to assess air infiltration in building envelopes. International workshop: Quality of Methods for Measuring Ventilation and Air Infiltration in Buildings, Proceedings (pp. 135-142). Presented at the International workshop: Quality of Methods for Measuring Ventilation and Air Infiltration in Buildings, Air Infiltration and Ventilation Centre (AIVC). 